\title{
A Multi-agent Approach for Range Image Segmentation
}

\author{
Smaine Mazouzi ${ }^{1}$, Zahia Guessoum ${ }^{2}$, Fabien Michel $^{1}$, and Mohamed Batouche ${ }^{3}$ \\ ${ }^{1}$ MODECO-CReSTIC, Université de Reims, B.P. 1035, 51687, Reims, France \\ \{mazouzi,fmichel\}@leri.univ-reims.fr \\ ${ }^{2}$ LIP6, Université de Paris 6, 104, av. du Président Kennedy, 75016, Paris, France \\ zahia.guessoum@lip6.fr \\ 3 Département d'informatique, Université de Constantine, 25000, Algérie \\ batouche@wissal.dz
}

\begin{abstract}
This paper presents and evaluates a multi-agent approach for range image segmentation. A set of reactive and autonomous agents perform a collective segmentation by partitioning a range image in its different planar regions. The agents move over the image and perform cooperative and competitive actions on the pixels, allowing a robust region extraction, and an accurate edge detection. An artificial potential field, created around the pixels of interest, ensures the agent coordination. It allows the agents to concentrate their actions around the edges and the noise regions. The experimental results show the potential of the proposed approach for scene understanding in range images, regarding both segmentation efficiency, and detection accuracy.
\end{abstract}

Keywords: Image segmentation, Multi-agent systems, Range image, Agent coordination, Artificial potential field.

\section{Introduction}

Image segmentation consists in assigning the pixels of an image to homogenous and disjoint subsets, providing a compact and convenient description of the image. In range imagery, segmentation algorithms can be divided into two dual categories: edge-based segmentation algorithms and region-based segmentation algorithms. In the first category, pixels that correspond to discontinuities in depth or in surface normals are selected, chained and then used to delimit the regions in the image [67. Region-based methods use geometrical surface descriptors to group pixels, with the same proprieties, in disjoint regions [8 10 3] . In both categories, algorithms must deal with noisy and uncertain data.

To overcome this difficulty, some authors have proposed multi-agent systems for 2-D image segmentation. Solutions provided by such systems inherit the advantages of the agent-oriented approach for collective problem solving. In such systems a single agent has a limited perception and limited capabilities, and it is not designed to solve an entire problem. Agents cooperate thus in order to provide a collective solution. Contrary to conventional systems, solutions in multi-agent systems emerge from the collective action of interactive agents [9]. 
In this paper, a new multi-agent approach for range image segmentation is presented and discussed. It consists in the use of reactive agents, which move over the image, and act on the visited pixels. While moving over the image, an agent adapts to the planar region on which it moves, and memorizes its proprieties. At the boundaries between regions, the agents will be in competition to align the pixels of the boundaries to their respective regions. The resulting alternative alignment of the boundary pixels preserves the region boundaries against smoothing. Noise regions, which are characterized by small sizes or by aberrant depths (outliers), prevent agents from adapting. So, the pixels on their borders are continuously aligned to the true regions that surround them. After several iterations these regions will entirely disappear.

This work aims to overcome the difficulty related to the local perception around the processed pixel. A pixel is therefore processed according to both its neighborhood, and the agents that visit this pixel. An agent acts on the pixels with more certainty, acquired from its move on large areas on the image regions. The combination of the global information memorized within the agent, and the local information of the image, provides more reliable decisions. We show in this work that despite the simplicity of the model used to represent the image surface, the obtained results are better than those provided by conventional approaches. We believe that interactions between agents provide an alternative way for image segmentation to that of approaches based on complicated and costly models. Extensive experiments have been performed using real images from the ABW database [5]. The obtained results show the high potential of the proposed approach for an efficient and accurate segmentation of range images.

The remainder of the paper is organized as follows: In Section 2, we review some agent-based approaches for image segmentation. Section 3 is devoted to the proposed approach. It describes the behavior of the agents, and shows the underlying collective mechanism to deal with the edge detection and the noise removal. The experimental results are introduced in Section 4, in which we discuss the parameter selection, and we analyze and comment the obtained results. Finally, a conclusion summarizes our contribution.

\section{Related Work}

Several agent-based systems have been proposed to deal with various problems in image analysis and object recognition. In this review we consider only some works that have addressed a solution in image segmentation.

Liu et al. [1] introduce a reactive agent-based system for brain MRI segmentation. Four types of agents are used to label the pixels of the image according to their membership grade to the various regions. In this system, the agents neither interact directly between them nor act on the image. Their actions depend only on their local perception. Nevertheless, each agent is created so that it becomes more likely to meet more homogenous pixels. For the same type of images, Richard et al. 12 propose a hierarchical architecture of situated and cooperative agents. Several types of agents were used. Interaction between agents 
in the various hierarchic levels has allowed to deal with the control over the low-level segmentation tasks. However, the system was specially optimized to brain MRI segmentation. The two previous systems can provide correct results because region characteristics are regular in the various brain anatomic parts. In addition, most of the edges in such images are jump edges (at discontinuities of image data), which are easy to detect, compared to roof or smooth edges (edges respectively at normal or curvature discontinuities).

Based on a cognitive architecture, Bovenkamp et al. 2] have developed a multi-agent system for Intra Vascular Ultra Sound (IVUS) image segmentation. They aim to elaborate a high knowledge-based control over the low-level image processing algorithms. In this system, an agent is assigned to every expected object in the image. In this work, the problem of the control over the segmentation algorithms seems to be well resolved. However, no agent or even behavior has been proposed to deal with uncertain and noisy data.

The proposed agent-based systems for image segmentation are specific to image contents. Following a supervised approach, these systems segment images in known and previously expected regions. The system proposed in this paper claims to be general and unsupervised. It aims to segment an image into its different regions by using some invariant surface proprieties. The adaptive and competitive behavior of the agents allows overcoming the constraint related to the restriction of the treatments to the local neighborhood of the pixels.

\section{Multi-agent Range Image Segmentation}

A range image is a discretized two-dimensional array where at each pixel $(x, y)$ is recorded the distance $Z(x, y)$ between the range finder and the corresponding point of the scene. A new image $Z^{*}$, called plane image is derived from the range image. Each pixel $(x, y)$ of the new image records the tangent plane to the surface at $(x, y)$. The tasks performed by the agents on the plane image are based on the comparison of planes. So, we consider that two planes $a x+b y+c z=d$ and $a^{\prime} x+b^{\prime} y+c^{\prime} z=d^{\prime}$ are equal if they have, according to given thresholds $\left(\operatorname{Tr}_{\theta}\right.$, $\left.\operatorname{Tr}_{D}\right)$, the same orientation, and the same distance to the coordinate origin. $\operatorname{Tr}_{\theta}$ and $\operatorname{Tr}_{D}$ are respectively the angle and the distance thresholds.

The plane image $Z^{*}$ is considered as the environment in which the agents are initialized at random positions. An agent checks if it is situated on a planar region, and adapts to this region if it is planar, by memorizing its plane equation. Next, the agent performs actions, which depend on both its state and the state of the pixel on which it is located. At each time $t$, an agent is characterized by its position $\left(x_{t}, y_{t}\right)$ over the image, and by its ability $A_{t}$ to act on the encountered pixels. At the beginning of the process, all the agents are unable to alter any pixel of the image. After having been adapted to a planar region, an agent becomes able to modify the first encountered pixel that not belongs to the current region ( $A_{t}=$ true). When an agent alters a pixel, it loses its alteration ability $\left(A_{t}=\right.$ false) and starts again searching for a new planar region. An agent having modified a pixel records in an appropriate two-dimensional array $I$, at $\left(x_{t}, y_{t}\right)$ the last state 
of the visited pixel: $I\left(x_{t}, y_{t}\right) \in\{$ smoothed, aligned, unchanged $\}$. We show next, that this simple behavior of the agents allows both the detection of the image edges, and the removal of the noise regions.

\subsection{Agent Behavior}

An agent adapts to the region of the image on which it is moving by computing and memorizing the proprieties of this region, and by adopting the suited behavior to the local image data. An agent can be in one of the following situations : searching for a planar region, moving on a planar region, aligning images pixels.

After its creation, an agent randomly moves within the image and searches for a planar region around its current position. The agent uses a region seed formed by the last $L$ visited pixels. $L$ is called the adaptation path-length. It represents the confidence degree that the agent is situated within a planar region. So, the agent considers that it is within a planar region if the pixels of the seed form a planar surface. The agent memorizes the new region and considers it as its current planar region. It becomes then able to alter the first encountered pixel that does not belong to its new region $\left(A_{t}=\right.$ true).

While moving inside a planar region, an agent smoothes the pixel on which it is located, by updating the equations of both the memorized plane and the plane at the position of the pixel. This is done by replacing the two equations by their weighted average. Let $(a, b, c, d)$ and $\left(a^{\prime}, b^{\prime}, c^{\prime}, d^{\prime}\right)$ be the parameters respectively of the plane at the current pixel, and the memorized plane. Resulting parameters of the weighted average plane are obtained as follows:

$$
\left(a^{\prime \prime}, b^{\prime \prime}, c^{\prime \prime}, d^{\prime \prime}\right)=\frac{1}{1+l}\left(a+l a^{\prime}, b+l b^{\prime}, c+l c^{\prime}, d+l d^{\prime}\right)
$$

where $l$ is the length of the path crossed by the agent on the planar region.

When an agent meets a pixel of interest (not belonging to its current planar region, i.e. noise pixels or edge pixels), the pixel is partially aligned to the planar region on which the agent moves. The parameters $\left(a^{\prime \prime}, b^{\prime \prime}, c^{\prime \prime}, d^{\prime \prime}\right)$ of the new plane equation at the pixel position are obtained by linear combination of the current parameters $(a, b, c, d)$ and the parameters of the memorized plane equation $\left(a^{\prime}, b^{\prime}, c^{\prime}, d^{\prime}\right)$ :

$$
\left(a^{\prime \prime}, b^{\prime \prime}, c^{\prime \prime}, d^{\prime \prime}\right)=\frac{1}{1+\xi}\left(a+\xi a^{\prime}, b+\xi b^{\prime}, c+\xi c^{\prime}, d+\xi d^{\prime}\right)
$$

where $\xi$ is the alteration strength.

The agent loses then its alteration ability $\left(A_{t}=\right.$ false $)$ and starts again to search for a new planar region. The alteration strength $\xi$ is a critical parameter, which affects the quality of the results and the time of computation. Indeed, high values of $\xi$ lead to a fast region detection. However, the resulting region boundaries are distorted and badly localized (Fig. 1b). Low values of $\xi$ result in a slow detection, but region boundaries in this case, are well detected and correctly localized (Fig.11). In order to speed up the segmentation process, without edge distortion, an agent chooses the alteration strength among $\xi_{\min }$ and $\xi_{\max }$ according to the information recorded by other agents in the array $I$. So, an agent assumes that 


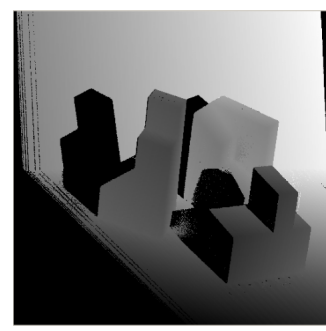

(a)

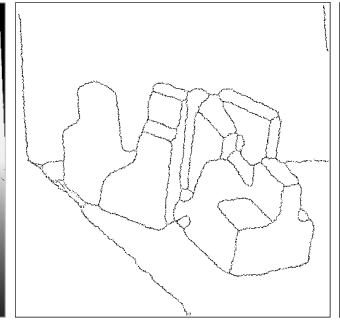

(b)

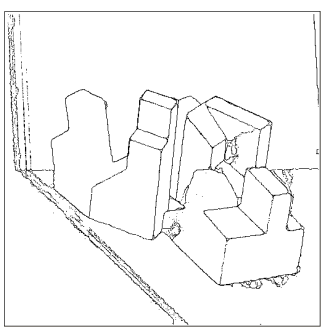

(c)

Fig. 1. The impact of the alteration strength on the segmentation results: (a) Range image (abw.test.8); (b) Segmentation results with $\xi_{\min }=\xi_{\max }=4$ at $t=2500$; (c) Segmentation results with $\xi_{\min }=0.3$ and $\xi_{\max }=5$ at $t=13000$

the current planar region is adjacent to a noise region, and thus uses $\xi_{\max }$ as alteration strength, if the number of "unchanged" pixels (situated within a noise region) around the agent is greater than a given threshold (fixed to 3 in our experimentations). Indeed, pixels labeled "unchanged" in the adjacent region mean that this latter is a noise region for which agents have not adapted and consequently have not smoothed its pixels. Otherwise, the agent assumes that the current planar region is adjacent to another planar one, where other agents have labeled the pixels as "smoothed" or "aligned". In this case, the agent uses the alteration strength $\xi_{\text {min }}$.

\subsection{Agent Coordination by Artificial Potential Field}

To endow agents with a self-organization mechanism, an artificial electrostaticlike potential field is used. It is created and updated around the aligned pixels. It allows agents to be gathered around pixels of region boundaries, and concentrate their actions at these pixels. Contrary to other works, where the potential field is created at known positions of objects (goals and obstacles) 413], the potential field in our case results from the interaction of agents with the objects in the environment (pixels). The intensity $\Psi(x, y)$ of the potential field at position $(x, y)$ created by a set of $P$ pixels beforehand aligned $\left\{\left(x_{i}, y_{i}\right), i=1 . . P\right.$ $\wedge I\left(x_{i}, y_{i}\right)=$ aligned $\}$ is given by:

$$
\Psi(x, y)=\sum_{i=1}^{P} \frac{k}{\sqrt{\left(x-x_{i}\right)^{2}+\left(y-y_{i}\right)^{2}}}, k \in R^{+}
$$

where $k$ is the constant of the electrostatic force, set to 1 .

An agent which is able to alter pixels ( $A_{t}=$ true) and situated at position $\left(x_{t}, y_{t}\right)$ undergoes an attractive force $\vec{F}$. This force is expressed by the gradient vector of the potential field:

$$
\vec{F}= \begin{cases}-\vec{\nabla} \Psi\left(x_{t}, y_{t}\right) & \text { if } A_{t}=\text { true } \\ \overrightarrow{0} & \text { otherwise }\end{cases}
$$


So, the agent movements, which are stochastic in nature, are weighted by the attractive force applied by the potential field. Agents are influenced to head for the pixels of interest, while keeping random moves. The random component of the agent moves allows the exploration of all regions of the image.

A Relaxation mechanism of potential field is also introduced. It allows the agents gathered around pixels of interest to be released and thus to explore other regions of the image. Around a given pixel, the field intensity decreases after every alteration of this pixel. The equation of the relaxation dynamic is expressed as follows:

$$
\Psi_{t+1}(x, y)=\mu \times \Psi_{t}(x, y), \mu<1
$$

$\Psi_{0}(x, y)$ corresponds to the created field after the first alteration of the pixel. The constant $\mu$ set to 0.9 , represents the decrease rate of the field intensity. After several alignments of a given pixel, the field intensity around this pixel decreases, and tends to zero. This situation represents the final state of the process, after which the system can be stopped.

\subsection{Edge Detection and Noise Removal}

While moving over the image, an agent smoothes the pixels that approximately belong to its planar region and considers all other pixels as noise pixels. Among these latter, the agent systematically aligns the first encountered one to its current region. However, pixels on the boundaries of regions are true-edge pixels, and thus should not be aligned. Nevertheless, the competition between agents preserves these pixels against an inappropriate smoothing. Indeed, around an edge between two adjacent planar regions, two groups of agents are formed on the two sides of the edge. Each group is formed of agents passing from one region to the other. Agents of each group align the pixels of the edge to their respective region. So, the pixels of the edge are continuously swapped between the two adjacent regions. The resulting alternative alignment of edge pixels allows these pixels to remain emergent in the image. This pattern of competitive actions between agents allows the emergence of the edges in the image, whose detection is not coded in any agent, but results from the collective action of all the agents.

Unlike the true regions of the image, which remain preserved against erasing, the noise regions continuously narrow, and they finally disappear. The borders of these regions are continuously aligned to the true planar regions that surround them. An agent, having aligned a pixel that belongs to the border of a noise region, and having moved inside it, will not be able to adapt. Consequently, the agent cannot align any pixel when leaving the noise region. This occurs in two distinct situations: 1) when the region is planar but insufficiently large to allow agents to cross the minimal path-length $L$, necessary to be able to adapt; 2) when the region is sufficiently large but not planar, or made up of random depths (noise). In both situations, the agent leaves the noise region and will adapt inside the surrounding planar one. As a summary we can say that true regions have large sizes, sufficient to allow agents to adapt and then align the boundary pixels when leaving these regions. However, noise regions, which are 
non planar or having weak sizes, prevent agents from adapting. Consequently, agents will be unable to align pixels on the boundaries of these regions when leaving them. As a result, the borders of a noise region are continuously aligned from outside by including their pixels in the true surrounding regions. After several iterations, all the noise regions will be completely erased. At the end of the process, all the regions in the image are well delimited by the detected boundaries. A simple region growing, steered by the detected boundaries, allows extracting the regions of the image.

\section{Experimentation and Analysis}

We have used a well known dedicated framework for the evaluation of range image segmentation algorithms [5]. The framework allows to compare a machinegenerated segmentation (MS) with a manually-generated segmentation, supposed ideal and representing the ground truth (GT). The most important performance evaluation metrics are the numbers of instances respectively of correctly detected regions, over-segmented regions, under-segmented regions, missed regions, and noise regions. Region classification is performed according to a compare tool tolerance $T ; 50 \%<T \leq 100 \%$, which reflects the strictness of the classification. In our case, four methods, namely USF, WSU, UB and UE, cited in [5] are involved in the comparison.

For our method, named 2ARIS, for Agent-based Approach for Range Image Segmentation, six parameters should be set: $\xi_{\min }, \xi_{\max }, \operatorname{Tr}_{\theta}, \operatorname{Tr}_{D}, N$, and $L$. These parameters are divided into two subsets: 1) $\xi_{\min }, \xi_{\max }, \operatorname{Tr}_{\theta}$, and $\operatorname{Tr}_{D}$ representing respectively the two alignment strengths, the angle threshold, and the depth threshold. 2) $N$ and $L$ are respectively the number of agents, and the adaptation path-length. For the first parameter subset, 256 combinations namely $\left(\xi_{\min }, \xi_{\max }, \operatorname{Tr}_{\theta}, \operatorname{Tr}_{D}\right) \in\{0.5,0.3,0.1,0.05\} \times\{1.0,3.0,5.0,7.0\} \times$ $\{15,18,21,24\} \times\{12,16,20,24\}$ were run on the training images. The performance criterion for these parameters is the average number of the correctly detected regions, with the compare tool tolerance $T$ set to $80 \%$. The two alteration strengths $\xi_{\min }$ and $\xi_{\max }$ are set respectively to 0.3 and 5.0. The thresholds $\operatorname{Tr}_{\theta}, \operatorname{Tr}_{D}$ were respectively set to 21 , and 16 . Note that inappropriate values of $N$ and $L$ can result in a high rate of segmentation errors. Indeed, an insufficient number of agents $(N)$ lead to an under-processing of the image. So, resulting regions are deprived of a set of pixels, which should be included in these regions. A low value of the adaptation path-length $L$ leads to take into account small planar regions, which should be considered as noise regions. However, higher values of $L$ can lead to consider some true planar regions, which are insufficiently large, as noise regions (see section 3.3). In order to set the parameters $N$ and $L, 25$ combinations of these parameters, namely $(N, L) \in\{1500,2000,2500,3000,3500\} \times\{3,5,7,9,11\}$ were run on the training set. In this case, the performance criterion is the average number of noise regions, with the compare tool tolerance set to $80 \%$. Obtained optimal values of $N$ and $L$ are respectively 2500 and 7 . 

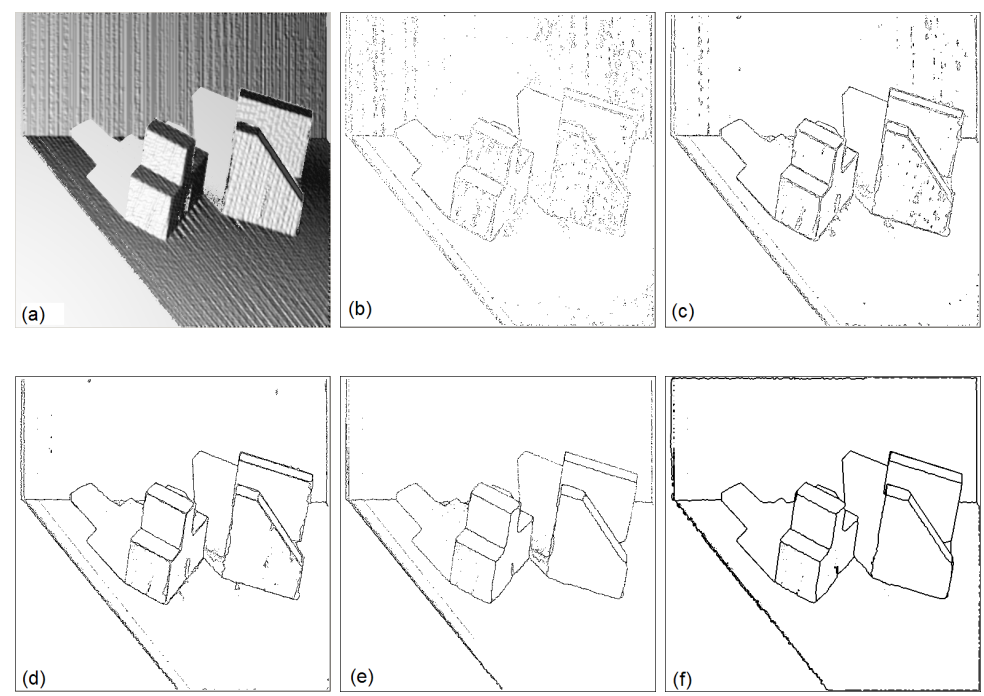

Fig. 2. Segmentation progression. (a) Rendered range image (abw.test.6); (b) at $t=1000$, (c) at $t=5000$; (d) at $t=9000$; (e) at $t=13000$; (f) Extracted regions.

Table 1. Average results of the different involved methods with $T=80 \%$

\begin{tabular}{ccccccc}
\hline Method & GT & Correct & Over-seg & Under-seg & Missed & Noise \\
\hline USF & 15.2 & 12.7 & 0.2 & 0.1 & 2.1 & 1.2 \\
WSU & 15.2 & 9.7 & 0.5 & 0.2 & 4.5 & 2.2 \\
UB & 15.2 & 12.8 & 0.5 & 0.1 & 1.7 & 2.1 \\
UE & 15.2 & 13.4 & 0.4 & 0.2 & 1.1 & 0.8 \\
2ARIS & 15.2 & 13.0 & 0.5 & 0.1 & 1.4 & 0.9 \\
\hline
\end{tabular}

Fig. 2 shows an instance of segmentation progression within time. The time $t$ represents the number of steps performed by each agent since the beginning of the process. Displaying a range image by a simple rendering algorithm (Fig. 2 a), allows to notice the high level of noise in the used images. Figures $2 \mathrm{~b}, 2 \mathrm{c}, 2 \mathrm{~d}$ and 2 s show the set of pixels of interest (edge or noise pixels) respectively at $t=1000,5000,9000$ and 13000. Regions are progressively smoothed by aligning the noise pixels to the surrounding planar regions. Edges between adjacent regions are also progressively thinned. At the end of the process, region borders consist of thin lines of one pixel wide (Fig. 2r). Fig. 2f shows the segmentation result obtained by displaying the borders of the extracted regions.

Table 1 contains the average results obtained with all the test images, and for all the performance metrics. The compare tool tolerance was set to the typical value $80 \%$. By considering both the correct detection and the incorrect detection metrics, obtained results show the good efficiency of our method. For all the incorrect detection metrics (instances of Over-segmentation, Under-segmentation, Missed Region, Noise Region), our method has equivalent scores to those of UE 


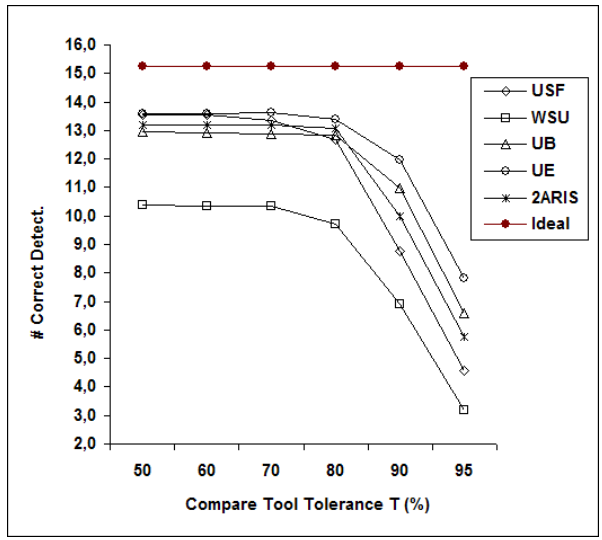

Fig. 3. Average results of correctly detected regions of all methods

and USF. The two latter scored higher than UB and WSU. Fig. 3 shows the average numbers of correctly detected regions for all the test images according to the compare tool tolerance $T$. Results show that the number of the correctly detected regions by our method is in average better than those of USF, UB and WSU. For instance, our method scored higher than WSU for all the values of the compare tool tolerance $T$. It scored higher than USF for $T \in\{80 \%, 90 \%$, $95 \%\}$, and better than UB for $T \in\{50 \%, 60 \%, 70 \%, 80 \%\}$.

\section{Conclusion}

In this paper, we have introduced a multi-agent approach for range image segmentation. Competitive actions between agents have allowed the emergence of the edges in the image. Image edges, for which no explicit detection was coded in any agent, result from the collective action of all the agents. Obtained results are better than those provided by the traditional algorithms. Moreover, used agents are weakly coupled and indirectly communicate via the environment (image). This allows parallel or distributed implementations, suited for a high computational efficiency. The experimental results obtained with real images from the ABW database show the potential of the proposed method for an efficient and accurate segmentation of range images. The average run time is $8 \mathrm{sec}$., on a Compaq PC $\mathrm{n} \times 8220$. The recorded run times were better that those provided by region based methods, and equivalent to edge-based ones. The proposed method should record interesting run times, when implemented on massively parallel architectures, such as Transputer-based architectures. The proposed approach can be extended to deal with more complex surfaces by defining their specific proprieties, and endowing the agents with the appropriate behavior. 


\section{References}

1. Bab Hadiashar, A., Gheissari, N.: Range image segmentation using surface selection criterion. IEEE Transactions on Image Processing 15(7), 2006-2018 (2006)

2. Bovenkamp, E.G.P., Dijkstra, J., Bosch, J.G., Reiber, J.H.C.: Multi-agent segmentation of IVUS images. Pattern Recognition 37(4), 647-663 (2004)

3. Ding, Y., Ping, X., Hu, M., Wang, D.: Range image segmentation based on randomized hough transform. Pattern Recognition Letters 26(13), 2033-2041 (2005)

4. Ferber, J.: Multi-Agent Systems: An Introduction to Distributed Artificial Intelligence. Addison-Wesley Longman Publishing Co., Inc., Boston, MA, USA (1999)

5. Hoover, A., Jean-Baptiste, G., Jiang, X., Flynn, P.J., Bunke, H., Goldgof, D.B., Bowyer, K.W., Eggert, D.W., Fitzgibbon, A.W., Fisher, R.B.: An experimental comparison of range image segmentation algorithms. IEEE Transactions on Pattern Analysis and Machine Intelligence 18(7), 673-689 (1996)

6. Inokuchi, S., Nita, T., Matsuda, F., Sakurai, Y.: A three dimensional edge-region operator for range pictures. In: 6th International Conference on Pattern Recognition, Munich, pp. 918-920 (1982)

7. Jiang, X., Bunke, H.: Edge detection in range images based on Scan Line approximation. Computer Vision and Image Understanding 73(2), 183-199 (1999)

8. Kang, S.B., Ikeuchi, K.: The complex EGI: A new representation for 3-D pose determination. IEEE Transactions on Pattern Analysis and Machine Intelligence 15(7), 707-721 (1993)

9. Krishnamurthy, E.V., Murthy, V.K.: Distributed agent paradigm for soft and hard computation. Journal of Network and Computer Applications 29(2), 124-146 (2006)

10. Li, S., Zhao, D.: Gradient-based polyhedral segmentation for range images. Pattern Recognition Letters 24(12), 2069-2077 (2003)

11. Liu, J., Tang, Y.Y.: Adaptive image segmentation with distributed behavior-based agents. IEEE Transactions on Pattern Analysis and Machine Intelligence 21(6), 544-551 (1999)

12. Richard, N., Dojat, M., Garbay, C.: Automated segmentation of human brain MR images using a multi-agent approach. Artificial Intelligence in Medicine 30(2), 153 176 (2004)

13. Tsuji, T., Tanaka, Y., Morasso, P., Sanguineti, V., Kaneko, M.: Bio-mimetic trajectory generation of robots via artificial potential field with time base generator. IEEE Transactions on Systems, Man, and Cybernetics, Part C 32(4), 426-439 (2002) 